\title{
Determination of thermal conductivity coefficient by Green-Kubo formula using the minimum image method
}

\author{
K. Hyżorek ${ }^{1}$ ，K. Ciesielczyk ${ }^{2}$ ，K.V. Tretiakov ${ }^{1,3 *}$ \\ ${ }^{1}$ Institute of Molecular Physics \\ Polish Academy of Sciences \\ Smoluchowskiego 17/19 \\ 60-179 Poznań, Poland \\ 2 Poznań University of Technology \\ Jana Pawta II 24 \\ 60-965 Poznań, Poland \\ ${ }^{3}$ The President Stanistaw Wojciechowski State University \\ of Applied Sciences in Kalisz. \\ Nowy Swiat 4, 62-800 Kalisz, Poland \\ *E-mail: tretiakov@ifmpan.poznan.pl
}

Received: 03 June 2019; revised: 27 June 2019; accepted: 29 June 2019; published online: 30 June 2019

\begin{abstract}
The thermal conductivity coefficients of solid argon have been evaluated by equilibrium molecular dynamic simulations. A Lennard-Jones interatomic potential has been used to model the interactions between argon atoms. In simulations and calculations of the thermal conductivity by the Green-Kubo formula, the long-range interactions between atoms have been taken into account using the minimum image method (MIM). The study shows that there are no significant differences between the values of the thermal conductivity obtained by method using MIM and those coming from traditional Green-Kubo approach. Both experimental data and results of molecular dynamics simulations are also in agreement with the Klemens-Callaway model for the thermal conductivity based on the three-phonon Umklapp scattering.
\end{abstract}

Key words: the Green-Kubo method, thermal conductivity, solid argon, Lennard-Jones potential, Molecular Dynamics simulations

\section{INTRODUCTION}

The knowledge of thermal conductivity of various materials is important not only from the theoretical point of view but also for practical applications. There are several methods to determine the thermal conductivity coefficient using computer simulations [1]. One of the most widely used is the Green-Kubo method in Equilibrium Molecular Dynamics (EMD) simulations [2]. The thermal conductivity and general expressions of other transport coefficients in bulk limit has been given by Kubo and Zwanzig [3, 4]. This method is based on analysis of the autocorrelation function of the microscopic heat current derived by Kirkwood [5]. Usually in calculations by the Green-Kubo formula, only nearest neighbors (or 1st and 2nd neighbors, etc.) interactions are considered directly [6] because accounting of the long-range interactions in simulations is "computationally expensive". So, final results are often corrected by the long-range corrections which are calculated using the mean-density approximation [1]. As a part of this approximation it is assumed that particles are distributed uniformly in space. This is a very good approach in the case of fluid but in the case of solid it is not always appropriate. It has been shown recently that the contribution of long-range interactions cannot be ignored in calculations of the elastic properties of the Lennard-Jones system [7]. In that work Tretiakov and Wojciechowski pro- 
posed a method for determining the elastic properties using the minimum image method (MIM) $[8,9]$ which allows one to take into account the contribution of long-range interactions directly. It has also been shown that elastic constants of the LJ system obtained (using the MIM) for such small systems as systems of about one hundred particles differ by less than $1 \%$ from that in the thermodynamic limit. On the other hand, it is known that the size of the system affects results of calculation of the thermal conductivity coefficient in bulk systems $[10,11]$. One of the potential reasons of the effect may be using the mean-density approximation of long-range interactions between particles.

In this work we evaluated the thermal conductivity of the Lennard-Jones system (on the example of solid argon) by the Green-Kubo approach using the minimum image method which allows us to take into account the long-range interactions between particles. We also compared the computed values with experimental data and with the hightemperature limit of the three-phonon scattering contribution to the thermal conductivity of solid argon.

\section{METHOD AND COMPUTATIONAL DETAILS}

In this study to describe interatomic interactions we used the Lennard-Jones (LJ) potential which has a form [1]

$$
\phi\left(r_{i j}\right)=4 \epsilon\left[\left(\frac{\sigma}{r_{i j}}\right)^{12}-\left(\frac{\sigma}{r_{i j}}\right)^{6}\right],
$$

where $\epsilon$ sets the energy scale, $\sigma$ is the atom diameter and $r_{i j}$ is the distance between atoms $i$ and $j$. A choice of LJ potential is not accidental. It is based on the fact that a use of this potential allows us to describe well some thermodynamical properties of noble gases, such as argon, kripton, xenon [2]. Here, we used the values of parameters for argon $\epsilon / k_{\mathrm{B}}=119.8 \mathrm{~K}$ and $\sigma=3.405 \AA$ [1]. In modern computer simulations, argon (which atoms' interactions modeled by the LJ potential) is often considered as a benchmark system to test new theories and computational methods including to better understand heat transfer in solid.

To calculate the thermal conductivity coefficient of bulk argon crystals we used the Green-Kubo formula [2]

$$
\lambda=\frac{1}{3 V k_{\mathrm{B}} T^{2}} \int_{0}^{\infty}\langle\boldsymbol{j}(0) \boldsymbol{j}(t)\rangle d t,
$$

where $T$ is temperature, $k_{\mathrm{B}}$ is the Boltzmann constant, $V$ is volume, and brackets denote the average over time. Factor $1 / 3$ corresponds to the fact that the diagonal components of the thermal conductivity tensor for cubically isotropic material are calculated. The microscopic heat current reads as $[5,6]$

$$
\boldsymbol{j}(t)=\sum_{i} \boldsymbol{v}_{i} \varepsilon_{i}+\frac{1}{2} \sum_{i} \sum_{j, i \neq j} \boldsymbol{r}_{i j}\left(\boldsymbol{v}_{i} \cdot \boldsymbol{F}_{i j}\right),
$$

where $\boldsymbol{v}_{i}$ is the velocity of particle $i, \boldsymbol{F}_{i j}$ is the force acting on atom $i$ due atom $j, m_{i}$ is the mass of atom $i$, and the microscopic site energy is

$$
\varepsilon_{i}=\frac{1}{2} m_{i}\left|\boldsymbol{v}_{i}\right|^{2}+u_{i} .
$$

In classical calculations of thermal conductivity using the Green-Kubo formalism, during simulation the LJ pair potential usually is cut off at some radius $\left(r_{\mathrm{c}}\right)$ and long-range corrections to the energy are calculated as follows [1]

$$
u_{\mathrm{LRC}}^{*}=\frac{8}{9} \pi \rho^{*}\left[\left(\frac{\sigma}{r_{\mathrm{c}}}\right)^{9}-3\left(\frac{\sigma}{r_{\mathrm{c}}}\right)^{3}\right],
$$

where $u^{*}=u / \epsilon$ is dimensionless energy, $\rho^{*}=(N / V) \rho^{3}$ is number density and $N$ is number of particles in the system. In conventional calculations of thermal conductivity, the contribution of long-range interactions to potential energy of the particle $i$ is taken into account through the energy correction $\left(u_{\mathrm{LRC}}^{*}\right)$ in the following way

$$
u_{i}=\sum_{j} \phi_{i j}+u_{\mathrm{LRC}}
$$

where the sum corresponds to the contribution of the particles in the simulation box for which $r_{i j}<r_{\mathrm{c}}$ (standard scheme [1]) and the second term is the correction of long-range interactions using the mean-density approximation (in this case Eq. (5)). Using the mean-density correction, information on the spatial arrangement of particles remaining outside the simulation box are lost.

Originally, the minimum image method was proposed by Ladd for calculations of the energy and pressure in systems with long-range interactions $[8,9]$. In the method, the calculations are performed for the $i$-th particle of coordinates $\left(x_{i}, y_{i}, z_{i}\right)$ and for the 26 images of the particle with coordinates $\left(x_{i}+k L, y_{i}+k L, z_{i}+k L\right)$ where $k=-1,0,1$ and $L$ is the size of the simulation box. In the proposed method, the contribution of nonuniform spatial arrangement of particles (concerns the crystal symmetry) to the microscopic flux energy due to considering the particle images is taken into account. Using the MIM, the potential energy of the particle $i$ is calculated as [7]

$$
u_{i}=\sum_{j} \phi_{i j}^{\mathrm{BOX}}+\sum_{k} \phi_{i k}^{\mathrm{MIM}}+u_{\mathrm{LRC}},
$$

where the first sum corresponds to the contribution of all particles in the simulation box, the second sum represents the contribution of the minimum image particles from outside of the simulation box. In the case, the cut-off radius of the LJ pair potential (in Eq. (5)) was taken from condition that the volume of sphere (with radius $r_{\mathrm{c}}$ ) equals the volume of cube $\left((3 L)^{3}\right)$ which considered in the MIM. 
Solid argon has the face-centered cubic (fcc) structure, with four atoms in the simple cubic cell. So, the studied systems consisted of $N=4 n^{3}(n=3,4,5,6)$ particles in periodic boundary conditions that resulting in the total numbers of particles considered in simulations using the MIM were 2916, 6912, 13500, 23328. The simulations were performed at temperature $T=75 \mathrm{~K}$ ( $T^{*}=0.626$ in reduced units) for 5 different molar volumes $V_{m}=21.92,22.37,23.24,24.00$ and $24.21 \mathrm{ml} / \mathrm{Mol}$ (which corresponds to the following densities $\rho=1.085,1.063,1.023,0.991,0.982 \mathrm{~kg} / \mathrm{m}^{3}$ of solid argon). These values were chosen to be comparable with available experimental and simulations data. For every phase point, 10 simulation runs with different initial velocities of particles were performed. The thermal conductivity coefficients were averaged from these 10 independent runs. The numbers of production simulation steps were $5 \cdot 10^{5}$ for two smallest systems and $10^{6}$ for larger systems after $10^{5}$ steps of equilibration. The time step was set to $\Delta t=0.002 \tau$, where $\tau=\sqrt{m \cdot \sigma^{2} / \epsilon}$. The Velocity Verlet integration algorithm was used to integrate the equations of motion $[1,2]$. Velocity scaling scheme $[1,2]$ was used to control the temperature. In classical calculations, the cut-off radius of the LJ pair potential was set to $r_{\mathrm{c}}=2.5 \sigma$. In simulations with the MIM, $r_{\mathrm{c}}=(3 L \sqrt[3]{3 /(4 \pi)}) \sigma$ was used.

\section{RESULTS AND DISCUSSION}

The size dependencies of thermal conductivity calculated by the Green-Kubo method with MIM are presented in Fig. 1. Tretiakov and Scandolo showed that the size dependence of the thermal conductivity coefficient is rather weak in EMD simulations using the Green-Kubo formalism [12]. In the present study we observe that size effects in determining the thermal conductivity with the Green-Kubo formula using the minimum image method are also negligible (Fig. 1). In further discussion we use the values of thermal conductivity obtained in thermodynamic limit $(N \rightarrow \infty)$ as it is shown in Fig. 1. As it can be seen in Fig. 2, the thermal conductivity coefficients of solid argon determined through MD simulations by the Green-Kubo formula using MIM are in good agreement with experimental data $[13,14]$ and previous simulation results [12].

Previously, [12] reported a good agreement both of simulation and experimental results with simplified approximation for the thermal conductivity in the high temperature limit which can be written as [15]

$$
\lambda=\frac{C}{a T \sqrt{m}} \frac{\left(\phi^{\prime \prime}\right)^{7 / 2}}{\left(\phi^{\prime \prime \prime}\right)^{2}},
$$

where $\phi^{\prime \prime}$ is the second derivative of the pair interaction potential and $\phi^{\prime \prime \prime}$ is the third derivative of the pair interaction potential with respect to the first-neighbor separation, $a$ is the lattice spacing of the primitive cubic cell, $m$ is the

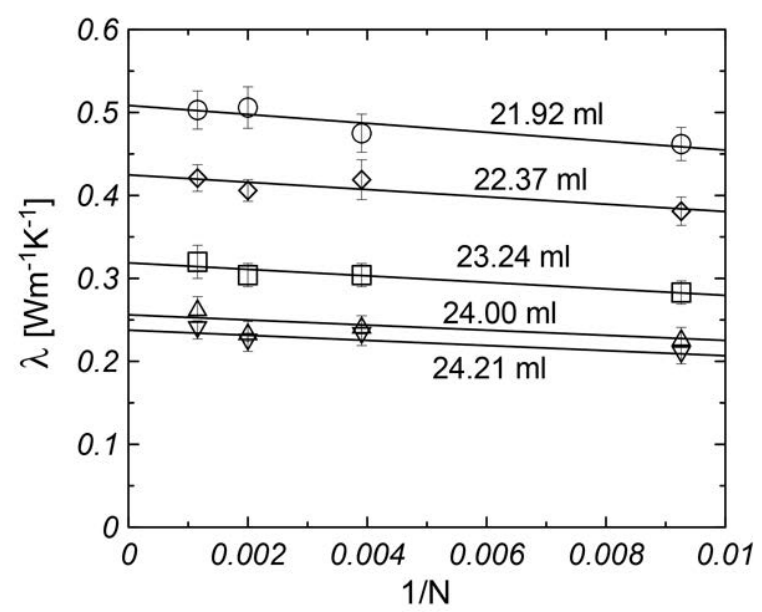

Fig. 1. Thermal conductivity coefficients of solid argon at $T=$ $=75 \mathrm{~K}$ for different molar volumes as a function $1 / N$. The solid lines are the linear fits to simulation data

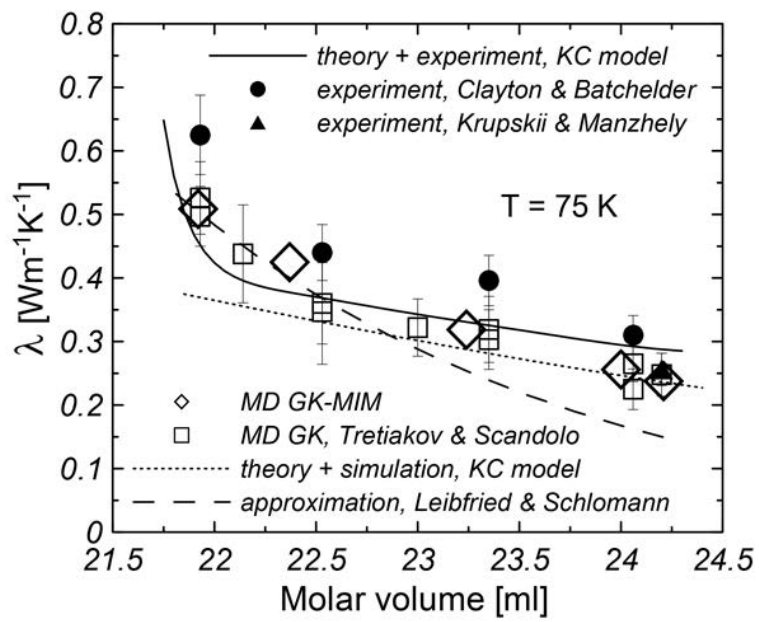

Fig. 2. Dependence of the thermal conductivity of solid argon on the molar volume at $75 \mathrm{~K}$. Full symbols represent experimental data. The black triangle is the Krupskii and Manzhelii result Ref. [13]. Full circles are the data of Clayton and Batchelder Ref. [14]. Open squares are Tretiakov and Scandolo results obtained by MD simulations using the Green-Kubo method Ref. [12]. Open diamonds represent the results of MD simulations by the Green-Kubo formula using MIM obtained in this work. The broken curve was calculated using Eq. (8). The solid line was calculated by Eq. (9) using experimental values of variables as initial data. The dotted line was also calculated by Eq. (9) but using data for a Lennard-Jones solid

atomic mass and $C$ is the quantity which involves constants and sums over nondimensional dispersion curves and anharmonic coefficients. At a fixed crystal volume, $C$ was evaluated for a LJ model and equal 8.12 [15].

In present work, we compare the obtained results with the Klemens-Callaway's (KC) model, where only acoustic phonon relaxation in resistive processes, such as three-phonon Umklapp scattering is taken into account. The Klemens-Callaway's expression for the lattice thermal conductivity in bulk is written as $[16,17]$ 


$$
\lambda=\left(\frac{k_{\mathrm{B}}}{\hbar}\right)^{3} \frac{k_{\mathrm{B}} T^{3}}{2 \pi^{2} v} \int_{0}^{\theta_{\mathrm{D}} / T} \frac{\tau_{\mathrm{U}} \chi^{4} e^{\chi}}{\left(e^{\chi}-1\right)^{2}} d \chi,
$$

where $\theta_{\mathrm{D}}$ is the Debye temperature (for argon $\theta_{\mathrm{D}}=92 \mathrm{~K}$ ), $v$ is the acoustic phonon group velocity, $\hbar$ is the Planck constant, and $\chi=\hbar \omega /\left(k_{\mathrm{B}} T\right)$, and $\omega$ is the phonon frequency. The relaxation time $\left(\tau_{\mathrm{U}}\right)$ for three-phonon Umklapp scattering in the high temperature limit was given by Klemens as $[16,18]$

$$
\tau_{\mathrm{U}}=\frac{\mu V_{0} \hbar \omega_{\mathrm{D}}}{2 \gamma^{2}\left(k_{\mathrm{B}} T\right)^{3} \chi^{2}},
$$

where $\mu$ is the shear modulus, $\omega_{\mathrm{D}}$ is the Debye frequency, $V_{0}$ is the volume per atom, and $\gamma$ is the Grüneisen parameter. In the considered model the thermal conductivity is dominated by the contribution of acoustic phonons, and we approximated the acoustic phonon group velocity by the average sound velocity [19]

$$
v_{s}=\left(\frac{1}{3}\left(\frac{1}{v_{\mathrm{L}}^{3}}+\frac{2}{v_{\mathrm{T}}^{3}}\right)\right)^{-\frac{1}{3}},
$$

where $v_{\mathrm{L}}$ is the longitudinal average sound velocity and $v_{\mathrm{T}}$ is the transverse average sound velocity.

The thermal conductivity of solid argon (by Eqs. (9) and (10)) has been calculated using two sets of parameters and physical quantities. In the first approach, we calculated the thermal conductivity using only experimental values of parameters and physical quantities which are in Eqs. (9)-(11). Here, the values of longitudinal and transverse average sound velocities, the Grüneisen parameter, and the shear modulus were taken from measurements by Keeler and Batchelder [20]. The solid line in Fig. 2 represents the result of this calculation. Good agreement results of $\mathrm{KC}$ model and experimental values of thermal conductivity of solid argon should be noted.

In the second approach, we used theoretical value of the Grüneisen parameter $\left(\gamma=-\frac{1}{6} \frac{\phi^{\prime \prime \prime} r_{0}^{2}+2\left[\phi^{\prime \prime} r_{0}-\phi^{\prime}\right]}{\phi^{\prime \prime} r_{0}+2 \phi^{\prime}}\right.$ where $r_{0}$ is the equilibrium distance [21]) and the elastic constants obtained by computer simulations [7] for calculation of the values of longitudinal and transverse average sound velocities $\left(v_{\mathrm{L}}=\sqrt{\frac{C_{11}+2 C_{12}+4 C_{44}}{3 \rho}}\right.$ and $v_{\mathrm{T}}=\sqrt{\frac{C_{44}}{\rho}}$ where $C_{i j}$ are the elastic constants). The dotted line in Fig. 2 show the thermal conductivity of solid argon estimated using parameters and physical quantities for the Lennard-Jones model. In this case we observe very good agreements between theoretical value of the thermal conductivity (Eq. (9)) and the thermal conductivity of the Lennard-Jones crystal obtained by computer simulations using the Green-Kubo method.

\section{SUMMARY}

The Molecular Dynamics simulations of solid argon with a Lennard-Jones model have been performed to determine the thermal conductivity by the Green-Kubo formula using the minimum image method. These studies show that when determining the thermal conductivity, the information on the spatial arrangement of particles remaining outside the simulation box is no such crucial, as distinct from the case of determination of elastic constants. This may be due to the fact that elastic constants are the second derivatives of the free energy with respect to the deformation and strongly depend on the symmetry of the crystal. The obtained results indicate that taking into account long-range interactions using MIM does not affect the calculated value of the thermal conductivity coefficient of solid argon. The obtained results showed, in agreement with previous work [12], that size effects in the determination of the thermal conductivity with the GreenKubo method are negligible.

The Klemens-Callaway model for the thermal conductivity based on the three-phonon Umklapp scattering reproduces both experimental data and results of molecular dynamics simulations rather well. It could mean that molecular dynamics simulations mimic well lattice dynamics, which results in correct description of the phonon scattering process at least in the high temperature regime.

The presented results confirm that molecular dynamics simulations with proper interatomic potential and the GreenKubo method is a very powerful calculation tool to evaluate the thermal conductivity of crystals.

\section{Acknowledgment}

This work was partially supported by the grant 2017/ 27/B/ST3/02955 of the National Science Centre, Poland. The computations were partially performed at Poznań Supercomputing and Networking Center (PCSS).

\section{References}

[1] M.P. Allen, D.J. Tildesley, Computer Simulation of Liquids, J.W. Arrowsmith Ltd., Bristol, UK (1987).

[2] J.P. Hansen, I.R. McDonald, Theory of Simple Liquids, Academic, New York (2005).

[3] R. Kubo, Statistical-Mechanical Theory of Irreversible Processes. I. General Theory and Simple Applications to Magnetic and Conduction Problems, J. Phys. Soc. Japan 12, 570586 (1957).

[4] R. Zwanzig, Time-Correlation Functions and Transport Coefficients in Statistical Mechanics, Annu. Rev. Phys. Chem. 16, 67-102 (1965).

[5] J.G. Kirkwood, The Statistical Mechanical Theory of Transport Processes I. General Theory, J. Chem. Phys. 14, 180 (1946).

[6] D.M. Heyes, Transport-Coefficients of Lennard-Jones Fluids: A Molecular-Dynamics and Effective Hard-Sphere Treatment, Phys. Rev. B 37, 5677 (1988).

[7] K.V. Tretiakov, K.W. Wojciechowski, Quick and accurate estimation of the elastic constants using the minimum image method, Comput. Phys. Commun. 189, 77-83 (2015).

[8] A.J.C. Ladd, Monte-Carlo simulation of water, Mol. Phys. 33(4), 1039-1050 (1977). 
[9] A.J.C. Ladd, Long-range dipolar interactions in computer simulations of polar liquids, Mol. Phys. 36(2), 463-474 (1978).

[10] D.P. Sellan, E.S. Landry, J.E. Turney, A.J. McGaughey, C.H. Amon, Size effects in molecular dynamics thermal conductivity predictions, Phys. Rev. B 81, 214305 (2010).

[11] K. Hyżorek, K.V. Tretiakov, Thermal conductivity of liquid argon in nanochannels from molecular dynamics simulations, J. Chem. Phys. 144, 194507 (2016).

[12] K.V. Tretiakov, S. Scandolo, Thermal conductivity of solid argon for molecular dynamics simulations, J. Chem. Phys. 120(8), 3765-3769 (2004).

[13] I.N. Krupskii, V.G. Manzhelii, Multiphonon Interactions and the Thermal Conductivity of Crystalline Argon, Krypton, and Xenon, Sov. Phys. JETP 28, 1097 (1969).

[14] F. Clayton, D.N. Batchelder, Temperature and volume dependence of the thermal conductivity of solid argon, J. Phys. C: Solid State Phys. 6, 1213 (1973).
[15] D.K. Christen, G.L. Pollack, Thermal conductivity of solid argon, Phys. Rev. B 12, 3380 (1975).

[16] P.G. Klemens, [In:] Solid State Physics, edited by F. Seitz and D. Turnbull, Academic Press, New York, 1st Edition (1958).

[17] J. Zou, A. Balandin, Phonon heat conduction in a semiconductor nanowire, J. Appl. Phys. 89, 2932 (2001).

[18] P.G. Klemens, [In:] Chem. and Phys. of Nanostructures and Related Non-Equilibrium Materials, edited by E. Ma, B. Fultz, R. Shall, J. Morral, and P. Nash, Minerals, Metals, Materials Society, Warrendale, PA (1997).

[19] J.P. Poirier, Introduction to the Physics of Earth's Interior, Cambridge University Press, Cambridge (1991).

[20] G.J. Keeler, D.N. Batchelder, Measurement of the elastic constants of argon from 3 to $77 \mathrm{~K}$, J. Phys. C: Solid State Phys. 3, 510 (1970).

[21] A.M. Krivtsov, V.A. Kuzkin, Derivation of Equations of State for Ideal Crystals of Simple Structure, Mech. Sol. 46, 387 (2011).

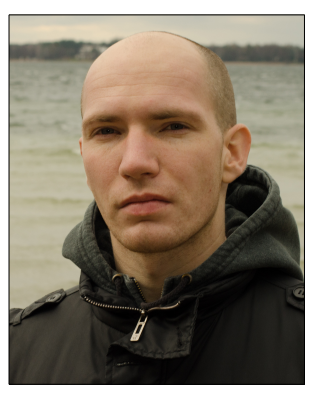

Krzysztof Hyżorek graduated in Technical Physics in 2014 from Poznań University of Technology. He received his Ph.D. degree in Physics from the Institute of Molecular Physics Polish Academy of Sciences (Poznań, Poland) in 2019, supervised by dr. habil. Konstantin Tretiakov. Dr. Hyżorek specializes in computer simulations, focusing his interest on transport properties of confined systems.

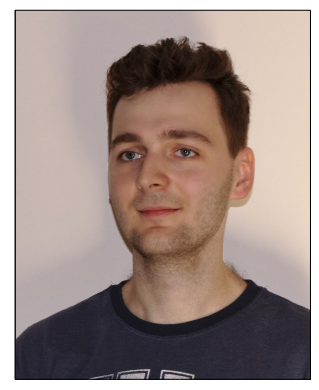

Karol Ciesielczyk earned his Bachelor of Engineering Degree in Technical Physics from Poznań University of Technology in 2016.

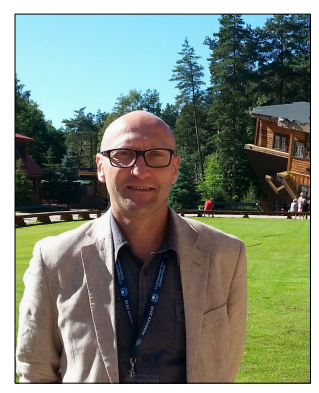

Konstantin V. Tretiakov graduated summa cum laude in Industrial Electronics from the Pavel Sukhoi State Technical University (Gomel, Belarus) in 1994. He received his Ph.D. degree in Physics from the Institute of Molecular Physics, Polish Academy of Sciences (Poznan, Poland) in 2000, supervised by Prof. Krzysztof Wojciechowski. In 2002-2004, Dr. Tretiakov was a Postdoctoral Fellow (with Prof. S. Scandolo) in the Condensed Matter and Statististical Physics Section at the Abdus Salam International Center for Theoretical Physics (Trieste, Italy). In 2007-2009, Dr. Tretiakov worked with Prof. Bartosz Grzybowski in the Department of Chemical and Biological Engineering at Northwestern University (Evanston IL, USA). Since 2014 he has been Associate Professor of Department of Computational Physics of Complex Systems in the Institute of Molecular Physics Polish Academy of Sciences. Dr. habil. Tretiakov specializes in statistical physics and computer simulation of many body systems, with research focusing on non-equilibrium self-assembling systems, transport properties of soft-matter, and elastic properties of solids, in particular materials exhibiting negative Poisson's ratio. 